\title{
Photometric study of the late-type contact binary YY Eridani ${ }^{\star}$
}

\author{
Y. Yang and Q. Liu \\ Yunnan Observatory, Chinese Academy of Sciences, Kunming, China \\ e-mail: bily@public.km.yn.cn
}

Received August 7; accepted December 1, 1998

\begin{abstract}
Complete light curves in $B$ and $V$ bands for YY Eridani are presented in this paper. The light curves appear to have a marked asymmetry with Max. I brighter than Max. II. Using the latest version of the WilsonDevinney light curve synthesis program, we have computed photometric solutions from the new light curves of YY Eri. The present solutions reveal that YY Eri is a $\mathrm{W}$ subtype contact binary. The asymmetries of the light curves are explained by the presence of dark spot on the primary or secondary component.
\end{abstract}

Key words: star: individual: YY Eri — binary: eclipsing

\section{Introduction}

YY Eri $\left(\mathrm{BD}-10^{\circ} 858=\mathrm{SAO} 149449, m_{v}=8.05-8.80\right.$, $p=0.3125$, sp. type G5V) was classified as a W UMatype binary by Bodokia (1938). The first photoelectric observations were made by Cilliě (1951); then it was observed again by Huruhata et al. (1953), Purgathofer et al. (1960), Budding (1983), Eaton (1985), Müyesseroğlu et al. (1990) and Maceroni et al. (1994). Light curve solutions were presented by Huruhata et al. (1953), Binnendijk (1965), Maceroni et al. (1982), Eaton (1986), Nesci et al. (1986), Maceroni et al. (1994) and Budding et al. (1997). The studies of the orbital period change were conducted by Cilliě (1951), Huruhata et al. (1953), Kwee (1958), Bhattacharyya (1967), Kim (1992) and Maceroni \& Van't Veer (1994). The spectrographic observations performed by Struve (1947) were re-analyzed by Huruhata et al. (1953). They determined a spectroscopic mass ratio of 0.59 , which is discrepant with the photometric mass ratio. This question was resolved by the new radial velocity study (Nesci et al. 1986).

Send offprint requests to: Y. Yang

* Tables 2 and 3 are only available in electronic form at the CDS via anonymous ftp to cdsarc.u-strasbg.fr (130.79.128.5) or via http://cdsweb.u-strasbg.fr/Abstract.html
The surface activity of the system was revealed by observations in various bands. Light curve asymmetry perturbations shifting in phase as evidence of photospheric spots (Maceroni et al. 1994) were exhibited by photometric observations. The emission lines as signatures of chromospheric activity were found in IUE spectra (Eaton 1983). And the system also appears to be an X-ray source in the survey of the Einstein satellite (Cruddace \& Dupree 1984).

\section{Observation}

Photoelectric photometry in $B$ and $V$ bands for YY Eri was conducted over four nights in November, 1984, with the 35-cm Cassegrain reflector of the Yunnan Observatory in China. A 1p21 photomultiplier was used in the observations. The two filters employed were the following: a Corning 5030 of stock thickness cemented with $2 \mathrm{~mm}$ Schott GG-13 for the blue region, and a Corning 3389 of Stock thickness for the yellow region. A circular diaphragm of $30^{\prime \prime}$ was used thoughout, and an integration time of 20 seconds for both stars and sky was chosen.

$\mathrm{BD}-11^{\circ} 831$ and $\mathrm{BD}-12^{\circ} 828$ were chosen as comparison star and check star, respectively, and measured alternatively between the observations of the binary. Nightly extinction coefficients were determined from observations of the comparison star, and the differential extinction correction were made. The observations were transformed into the $U B V$ system with the aid of six standard stars in the Pleiades during the period of observations. The magnitude differences between the check star and the comparison star were constant within probable errors of \pm 0.010 $\operatorname{mag}(V)$ and $\pm 0.012 \operatorname{mag}(B)$ during the observations.

From the observations, three times of primary minimum light and two times of secondary minimum light were derived by means of polynomial fitting and are listed in Table 1. Because of the complexities in the orbital period change of YY Eri, to combine the observations, we have adopted the light ephemeris found by least square 


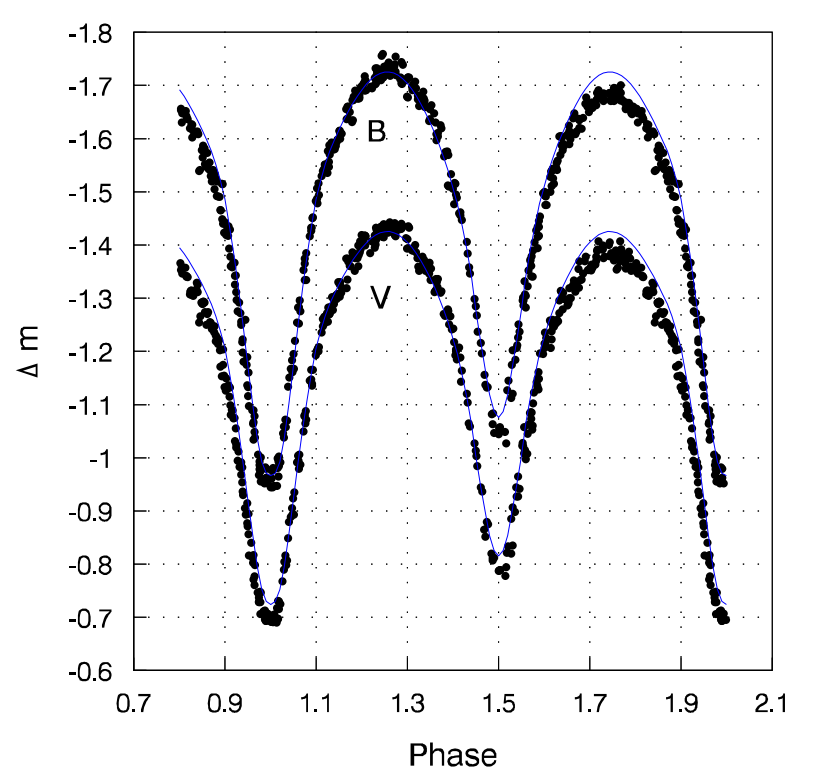

Fig. 1. Observed (points) and computed $B V$ light curves (solid lines) of YY Eri, assuming a standard photometric model. To enhance clarity, the two light curves have been displaced vertically by arbitrary amounts

Table 1. Minima of YY Eri

\begin{tabular}{llll}
\hline JD.Hel. & Min.-type & $E$ & $\mathrm{O}-\mathrm{C}$ \\
\hline $2446020+$ & & & \\
$6.1049(4)$ & II & -6.5 & +0.0004 \\
$6.2641(3)$ & I & -6 & -0.0013 \\
$7.2304(3)$ & I & -3 & +0.0000 \\
$8.1945(3)$ & I & 0 & -0.0008 \\
$8.3558(7)$ & II & 0.5 & -0.0003 \\
\hline
\end{tabular}

method from the minima in the Table 1 and the minima published by Marco et al. (1993) as follows:

Min.I $=\mathrm{JD}$ (hel.)2446028.1953+0.3216498 $\times E$.

In each colour 440 individual observations were secured. The magnitude differences in the sense of YY Eri minus $\mathrm{BD}-11^{\circ} 831$ together with their heliocentric Julian dates are listed in Table 2 for $V$ and Table 3 for $B$. The light curves of YY Eri are shown in Fig. 1 in solid points. All phase is covered at least twice. The light curves appear to exhibit a typical O'Connell effect, with Maximum I brighter than Maximum II by $0.042 \operatorname{mag}(V)$ and 0.048 $\operatorname{mag}(B)$ and Maximum I shifting to phase of 0.27 .

\section{Photometric solution}

Photometric solutions were obtained by means of the latest version of the WD program (Wilson 1992) which includes a new reflection treatment, the option of using nonlinear limb-darkening laws and the ability to adjust spot parameters. The individual observations were combined into 54 normal points in each colour and the two light curves were employed simultaneously in computing solutions. The convergence of the minimization procedure was obtained by means of the method of multiple subsets (Wilson \& Biermann 1976).

We adopted a temperature of $5600 \mathrm{~K}$ for star 1 (star eclipsed at Min. I), which corresponds to spectral type of G5V. The other adopted parameters were: Claret et al.'s (1990) values of the limb darkening coefficient $\left(x_{1}=x_{2}=\right.$ 0.84 for $B, 0.66$ for $V$ ), Lucy's (1967) values of the gravity darkening coefficient $\left(g_{1}=g_{2}=0.32\right)$ and Rucinski's (1969) values of the albedo $\left(A_{1}=A_{2}=0.50\right)$. Adjustable parameters were the orbital inclination, i, the mean temperature of star $2, T_{2}$, the potential of the components, $\Omega_{1}$ and $\Omega_{2}$, and the luminosity of star $1, L_{1}$ (the Planck function was used in computing the luminosity).

In order to search for an appropriate photometric mass ratio to compare with the spectroscopic mass ratio, solutions were made for a series of fixed values of the mass ratio $q=m_{2} / m_{1}(0.30,0.40,0.50,0.60,0.70,0.80,0.90,1.20$, $1.60,2.00,2.50$ and 3.00). Assuming a detached system initially, the differential corrections started from the mode 2 , but the converged solutions were always obtained at the contact mode 3 . The resulting sums, $\Sigma$, of the weighted square deviations of the converged solutions for each value of $\mathrm{q}$ are plotted in Fig. 2. The best fit is for $q=2.50$. At this point, the set of the adjustable parameters was expanded to include $q$. The mass ratio converged to a value of $q=2.36799$ in the final solution. This solution indicates that YY Eri is a W-type W UMa binary, in agreement with the results published by Binnendijk (1965), Maceroni et al. (1982), Nesci et al. (1986) and Maceroni et al. (1994). The photometric parameters are listed in Table 4, where star 1 indicates more massive component and star 2 is of less mass. The fit of the computed light curves is shown in Fig. 1 in solid lines.

While the overall fit of the computed light curves is quite satisfactory, Fig. 1 shows obvious distortions in the observed light curves that seems to be due to surface inhomogeneities of the components. Unequal quadrature light level, namely, the O'Connell effect, is known in many eclipsing binaries and several suggestions have been made to explain this effect by various authors. For YY Eri, the observed distortion, with Maximum II being fainter than Maximum I, may result from a cool region on either component. It has been assumed that the spot is on the star 1 or the star 2 and the several groups of dark spots or hot spots have been tested. While a converged solution with the hot $\operatorname{spot}(\mathrm{s})$ could be not found, the best fit to the observed light curves was found with dark spot on the star 1 or the star 2 . The solutions with the dark spot (co-latitude, $q_{\mathrm{s}}$, longitude, $f_{\mathrm{s}}$, angular radius, $r_{\mathrm{s}}$, all in degrees, and the temperature factor $T_{\mathrm{s}} / T_{*}$, with $T_{*}$ the local effective temperature of the surrounding photosphere) are also listed in Table 4. The solution labeled Dark 1 is with a dark spot on the primary (more massive) star and the solution labeled Dark 2 with a dark spot on the secondary 
Table 4. photometric solutions of YY Eri

\begin{tabular}{|c|c|c|c|c|c|c|}
\hline & & \multicolumn{2}{|l|}{ unspotted } & \multicolumn{3}{|c|}{ spotted } \\
\hline & & & & dark 1 & & dark 2 \\
\hline$q=m_{2} / m_{1}$ & & $0.4223(18)$ & & $0.4699(7)$ & & $0.4535(6)$ \\
\hline$i$ & & $81.76(35)$ & & $82.12(18)$ & & $82.42(20)$ \\
\hline$\Omega$ & & $2.6921(17)$ & & $2.7714(5)$ & & $207436(6)$ \\
\hline$f_{\mathrm{s}}$ & & - & & 64.60 & & 59.90 \\
\hline$q_{\mathrm{s}}$ & & - & & 83.70 & & 258.30 \\
\hline$r_{\mathrm{s}}$ & & - & & 21.10 & & 16.80 \\
\hline$T_{\mathrm{s}} / T_{*}$ & & - & & 0.770 & & 0.730 \\
\hline$\Sigma$ & & 0.008510 & & 0.001985 & & 0.001988 \\
\hline$f$ & & 2.66 & & 3.70 & & 3.41 \\
\hline$L_{1 B} / L_{t B}$ & & $0.6124(25)$ & & $0.5979(13)$ & & $0.6077(12)$ \\
\hline$L_{1 V} / L_{t V}$ & & $0.6285(19)$ & & $0.6123(10)$ & & $0.6216(9)$ \\
\hline star & 2 & 1 & 2 & 1 & 2 & 1 \\
\hline$T$ & 5600 & $5317(11)$ & 5600 & $5349(6)$ & 5600 & $5356(6)$ \\
\hline$r($ pole $)$ & $0.2940(9)$ & $0.4353(16)$ & $0.3041(4)$ & $0.4282(5)$ & $0.3008(4)$ & $0.4307(5)$ \\
\hline$r($ side $)$ & $0.3077(10)$ & $0.4657(22)$ & $0.3187(5)$ & $0.4574(6)$ & $0.3151(5)$ & $0.4603(7)$ \\
\hline$r($ back $)$ & $0.3456(17)$ & $0.4954(30)$ & $0.3576(8)$ & $0.4886(9)$ & $0.3538(9)$ & $0.4911(10)$ \\
\hline
\end{tabular}

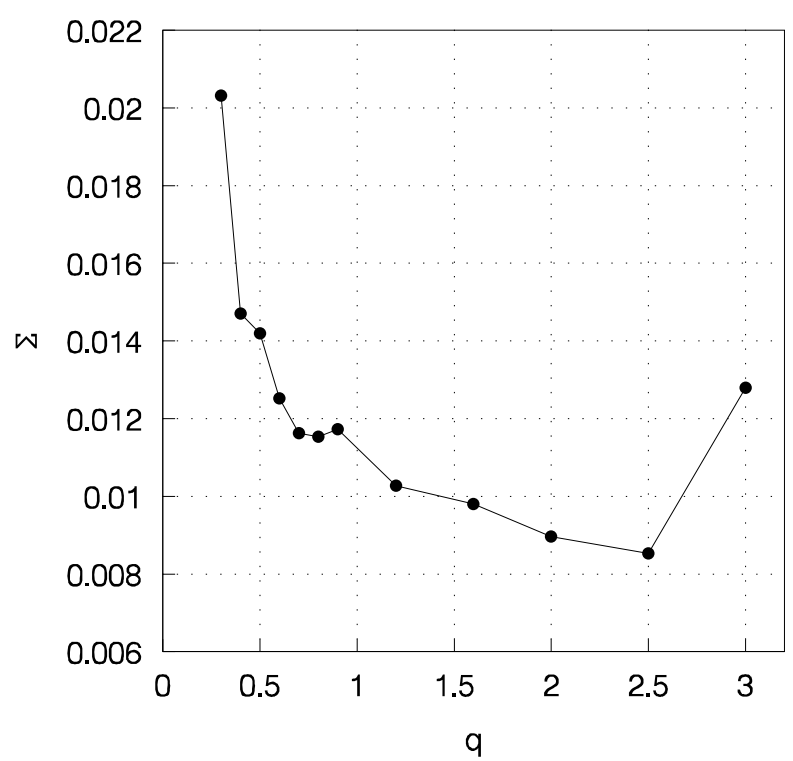

Fig. 2. Variance of the computed fit as a function of the mass ratio $q$

star. The solution labeled Dark 1 turns out to be slightly better quality than the other. The fit of the computed light curves corresponding to the solution in Table 5 with the dark spot on the primary star is shown in Fig. 3 in solid lines.

\section{Discussion}

The light curves of YY Eri have shown a pattern of variable O'Connell effect with some asymmetry, that is, the system tending to be somewhat brighter at its maximum I (after the primary minimum) than the following peak (maximum II). An inverse O'Connell effect (maximum II

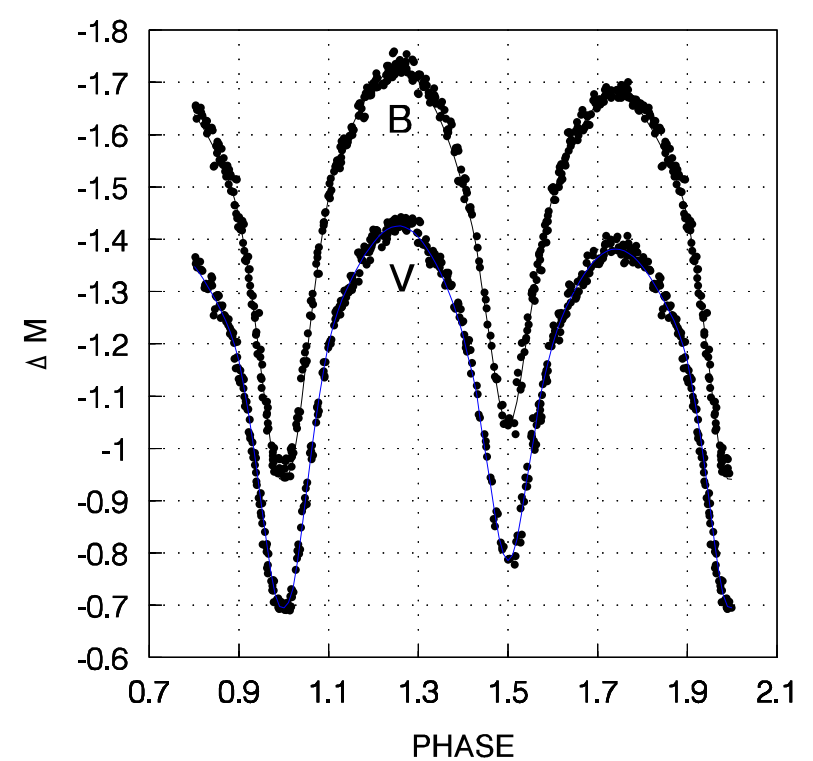

Fig. 3. As Fig. 1, but with dark spot included in the photometric model

brighter than maximum) can be seen in the observations of Müyesseroğlu et al. (1990). A figuration of the variable O'Connell effect in $V$ band for this system is shown in Fig. 4, which shows the observations carried out in 1958 by Purgathofer et al. (open circular points), in 1976 by Eaton (solid circular points), in 1984 by us (fork points), in 1988 by Müyesseroğlu et al. (solid rhomb points), and in 1990 by Maceroni et al. (open rhomb points). For comparison, all light curves were reduced assuming that they have the same magnitude difference at the phase of 0.5 . As shown in Fig. 4, the variation of the O'Connell effect of this system is very obvious and seems to be regular. The regularity of the variation becomes more obvious in 
Table 5. Parameters of the $V$ light curve properties of YY Eri

\begin{tabular}{cccccc}
\hline & 1958 & 1976 & 1984 & 1989 & 1990 \\
Max.I-Min.I & $0 . \mathrm{m} 718$ & 0.736 & $0 . \mathrm{m} 730$ & $0 . \mathrm{m} 685$ & 0.714 \\
Max.I-Min.II & 0.649 & 0.666 & 0.649 & 0.616 & 0.645 \\
Max.II-Min.II & 0.630 & 0.613 & 0.605 & 0.644 & 0.634 \\
Max.II-Min.I & 0.700 & 0.682 & 0.687 & 0.713 & 0.703 \\
Max.I-Max.II & 0.018 & 0.054 & 0.042 & -0.028 & 0.011 \\
mean Max.-Min.I & 0.709 & 0.709 & 0.709 & 0.699 & 0.709 \\
mean Max.-Min.II & 0.640 & 0.640 & 0.627 & 0.630 & 0.640 \\
\hline
\end{tabular}

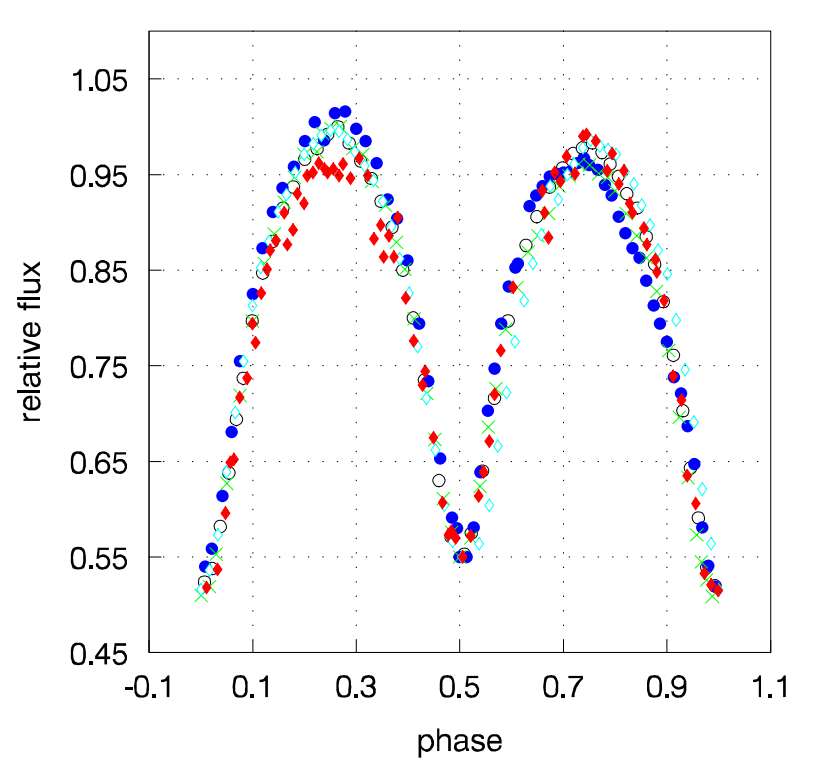

Fig. 4. Observed $V$ light curves of YY Eri from 1958 to 1990. To compare, all light curves have been reduced to the same magnitude level at 0.50 of phase

Table 5, in which some parameters of the light curves for YY Eri are listed.

Comparing the light curves with each other, we find that the rise of a light maximum level is always accompanied with the drop of the other light maximum level so that the mean deepness (average of the ascending and the descending branches) of the primary or secondary eclipse is always constant. In order to study physical implication of this phenomenon, it is necessary to investigate the variable O'Connell effect of more systems.

The photometric solutions of the light curves presented by different authors reveal a constant radius ratio and variable luminosity ratio of the two components for YY Eri. At least there are three sets of solutions obtained by using Wilson-Devinney procedure in the literatures. Some parameters of these solutions are listed in Table 6 , where the component radii, $r_{1}$ and $r_{2}$ are the geometrical mean of the polar, side and back radii, which were computed ad hoc with the WD code when not given in the original papers. $L_{1}$ and $L_{2}$ are relative luminosity of the primary and secondary component in $V$ band, respectively. Dark 1 indicates the solution with a cool spot on the surface of the
Table 6. Photometric parameters of YY Eri

\begin{tabular}{ccrlc}
\hline & $1958 N$ & $1984 Y$ & & $1990 M$ \\
$\Delta m$ & 0.02 & 0.04 & & 0.01 \\
& unspotted & unspotted & Dark 1 & Dark 1 \\
$L_{1}$ & 0.649 & 0.629 & 0.612 & 0.640 \\
$L_{2}$ & 0.351 & 0.371 & 0.388 & 0.360 \\
$L_{2} / L_{1}$ & 0.541 & 0.591 & 0.633 & 0.562 \\
$r_{1}$ & 0.468 & 0.470 & 0.458 & 0.458 \\
$r_{2}$ & 0.313 & 0.311 & 0.316 & 0.315 \\
$r_{2} / r_{1}$ & 0.669 & 0.662 & 0.690 & 0.688 \\
\hline
\end{tabular}

primary component. Column $\Delta m(=m(\max . \mathrm{I})-m(\max$. II)) in Table 6 gives a measure of the O'Connell effect (i.e. unequal height of the maxima) in $V$ color. The letter $N, M$ or $Y$ indicates the solution by Nesci et al. (1986), by Maceroni et al. (1994) or by us (the present paper), respectively. Comparing the unspotted solution of Nesci et al. with our unspotted solution, the values of $r_{2} / r_{1}$ from the two solutions are both equal to about 0.66 , but the value of $L_{2} / L_{1}$ from our solution is about $9 \%$ larger than that of Nesci et al.'s solution. Moreover, we notice that the difference of the O'Connell effect between the two light curves observed in 1984 and in 1958 is about 0.02 mag in $V$ band. Comparing our solution spotted on the primary component with that of Maceroni et al. also spotted on the primary component, again the values of $r_{2} / r_{1}$ of the two solutions are both equal to about 0.69 , whilst $L_{2} / L_{1}$ from our solution is about $13 \%$ larger than that of Maceroni et al. The difference of the measure of the O'Connell effect between the two light curves observed in 1984 and in 1990 is about $0.03 \mathrm{mag}$ in $V$ band. Therefore, we conclude that the variation of the O'Connell effect of YY Eri may be related with the variation of $L_{2} / L_{1}$, but is not related with the variation of $r_{2} / r_{1}$. This suggests that the variation of the O'Connell effect of this system is probably related with activity of spots on one or both components.

Acknowledgements. The authors would like to thank Dr. F. Van't Veer for his valuable comments and suggestions on the original version of this paper and Mr. Z. Zhang and Y. Zhang for their assistance in the observations. We would also like to express our gratitude for the support from the Chinese National Science Foundation. 


\section{References}

Bhattacharyya I.C., 1967, Kadaikanal Observatory Bull Ser., No. 181

Binnendijk L., 1965, AJ 70, 209

Bodokia V.M., 1938, Bull. Astrophys. Obs. Abastumani No. 3

Budding E., 1983, Inf. Bull. Var. Stars, No. 2300

Budding E., Kim C.-H., Demircan O., Myesseroglu Z., Saijo. K., Banks T., 1997, Astrophys. Space Sci. 246, 229

Cilliě G.G., 1951, Harvard Bull. No. 920, 41

Claret A., Gimenez A., 1990, A\&A 230, 412

Cruddace R.G., Dupree A.K., 1984, ApJ 277, 263

Eaton J.A., 1983, ApJ 268, 800

Eaton J.A., 1985, PASP 97, 896

Eaton J.A., 1986, Acta Astron. 36, 79

Huruhata M., Dambara T., Kitamura M., 1953, Tokio Ann. Sec. Ser. 3, 227

Kim C.H., 1992, in Kondo Y., Sistero R.F. and Polidan R.S. (eds.). IAU Symp. 151, p. 383
Kwee K.K., 1958, Bull. Astron. Inst. Nederlands 14, 131

Lucy L.B., 1967, Zert. Astrophys. 65, 89

Maceroni C., Milano L., Russo G., 1982, A\&AS 49, 123

Maceroni C., Van't Veer F., 1994, A\&Ap 289, 871

Maceroni C., Vilhu O., Van't Veer F., Van Hamme W., 1994, A\&A 288, 529

Marco A.H., Jose H.P., Rosario P., Manuel R.H., Manuel R.B., Eloy R., Omar L.C., 1993, IBVS 3935

Müyesseroğlu Z., Demircan O., Derman E., Selam S., 1990, in Active Close Binaries, Ibanoglu C. (ed.). Kluwer Acad. Publ. Dordrecht, p. 277

Nesci R., Maceroni C., Milano L., Russo G., 1986, A\&A 159, 142

Purgathofer A., Purgathofer I., 1960, Mitt. Wien 10, 195

Rucinski S.M., 1969, A\&A 19, 245

Struve O., 1947, ApJ 106, 92

Wilson R.E., 1992 (private communication)

Wilson R.E., Biermann P., 1976, A\&A 48, 349 